(RESEARCH ARTICLE)

\title{
Liver cell membrane antibody, kidney injury molecule and lungs tumour antigen expression on the MCF-7 cell induced breast cancer administered with Citrus limon juice and tamoxifen in Sprawgue dawley rats
}

\author{
Oyebadejo SA $1{ }^{*}{ }^{*}$, Nsuhoridem SA ${ }^{2}$ and Oyeleye $\mathrm{OE}^{3}$ \\ ${ }^{1}$ Department of Medical Laboratory sciences, Faculty of Allied Health Sciences, Kampala International University-Western \\ Campus, Ishaka, Bushenyi, Uganda. \\ ${ }^{2}$ Derindam Research Institute of Biotechnology, Uyo, Akwa-Ibom, Nigeria. \\ ${ }^{3}$ Department of Medical Laboratory sciences, School of Health Sciences, Univerisity and Strategic Institute of the Estuary \\ (IUEs/INSAM), Ndokoti, Douala, Cameroon.
}

Publication history: Received on 03November 2020; revised on 11December 2020; accepted on 13December 2020

Article DOI: https://doi.org/10.30574/wjarr.2020.8.3.0411

\begin{abstract}
Monitoring and evaluation have been one of the major measures to determine possible progression of response of disorder to the alternative treatments, however Anti-Liver Cell membrane Antibody, Kidney Injury Molecule and Lungs Tumour Antigen Expression on the MCF-7 Cell Induced Breast Cancer Rats administered with Citrus Limon juice and Tamoxifen were carried out, Over one hundred and twenty Sprague dawley rats of 40 days old average body weight 180-220g were divided into ten (10) containing of 12 animals per group, group 1 was control, fed only with rat chow and water, group 2 was MCF-7 cell line induced rats alone (BCIR only), group 3 Citrus Limon juice (CLJ) at 8.88\%, Group 4 Citrus Limon juice (CLJ) at 17.32\%, group 5 Citrus Limon juice (CLJ) at 25.98\%, group 6 was given $0.2 \mathrm{mg} / \mathrm{kg}$ of Tamoxifen alone, group 7 (BCIR+CLJ at 8.88\%), group 8 (BCIR+CLJ at 17.32\%), group 9 (BCIR+CLJ at 25.98\%) and group $(B C I R+0.2 \mathrm{mg} / \mathrm{kg}$ of Tamoxifen). Acute and sub - acute toxicity were carried out after the establishment of safety dose following determination of LD50, on the fresh Citrus Limon juice Breast cancer induction and Tumor sizes were analysed. At the end of the administration, animals were sacrificed, fresh blood was taken, centrifuged at $3000 \mathrm{rpm}$, serum was collected and stored at $8^{\circ} \mathrm{C}$ Breast Cancer Tumor Associated Assays, Kidney Injury, Liver cell Membrane Antibody and Lung Tumor Antigens were analysed. Result, Mammary tumor induction was succeeded in all the MCF-7 cell line induced groups with minimum number of morbidity. Tumor size was significantly increase in tumor mass BCIR only at P $>0.001$ when compared to BCIR + CLJ and BCIR + Tamoxifen groups. Immunoogical expression of Breast Cancer Associated Tumor revealed significant increase suppressor gene/protein, Immunocytochemical in-vivo of Breast Cancer Associated Organs showed significant increase in KIM, LCMA and LTA at P>0.001 in BCIR when compared to all the BCIR + CLJ, BCIR + Tamoxifen and other non-BCIR groups.

In conclusion, this study showed that KIM, LCMA and LTA exhibited strong Immunochemical expression of Citrus Limon juice potency possessing possible anticancer activities without posing pathological conditions on the body during and after usage, hence KIM, LCMA and LTA are demonstrated to be valuable in the monitoring and evaluation progression of tumor as Citrus Limon Juice could be used as alternative therapy in the treatment of Breast cancer that are hormonal dependant in similar manner to Tamoxifen.
\end{abstract}

Keywords: KIM; LCMA; LTA MCF-7 Cell lines; Breast cancer; Citrus Limon Juice; Tamoxifen; Sprawgue Dawley Rats

\footnotetext{
${ }^{*}$ Corresponding author: Oyebadejo S.A

Department of Medical Laboratory sciences, Faculty of Allied Health Sciences, Kampala International University-Western Campus, Ishaka, Bushenyi, Uganda.
} 


\section{Introduction}

Breast Cancer Associated Organ are generally considered as metastasis occurring in line with Breast cancer in some vital organs considered to play important role in the management of Breast cancer, such organs include Liver, Kidneys and Lungs as a result of their role in maintaining normal body functions. Breast Cancer usually claim life of victim not a single mechanism and due to impairment rendered on vital organs, thus make the manifestation to be more difficult to be controlled especially if adequate measure is not taken at the right time [1]. Liver metastasis has been documented to occur at the rate of 5-12\% in 50\% of patients suffering from Breast Cancer which occur as impairment of liver function thereby endangering the life of the patients [7]. Renal failure has been known to be a difficult commodity in Breast Cancer patients as well due to the kidney metabolizes of most chemotherapeutic drugs as a result of impairment in renal function capacities [8]. Distant metastasis may occur in the lungs as $60 \%$ of the Breast cancer patients were reported to develop tumor in the lungs resulting in the spread of malignant cells into the Lungs [9]. The use of Immunological expression in monitoring and evaluation is of great important tools in tumor progression, Kidney Injury Molecules, KIM is a type of membrane protein containing extracellular and cytoplasmic portion which are of T-cell immunoglobulin mucin receptors express in the kidney and other tissue due to infections, autoimmune responses or damaging in the kidney damages which signify onset and indicator for monitoring and evaluating of kidney abnormalities in breast cancer metastasis which are likely to affect the kidney [10]. Liver cell membrane Antibody (LCMA) is the protein of parenchymal and non-parenchymal cell of the liver that are expressed or produced during disturbance to the liver cells, or injury [3], meanwhile [12] Anti lung tumor antigen (LTA) as a protein belonging to the family of tumor necrosis factor, cytokine like in nature produced by lymphocytes, it is inducible, secreted and form anetertotrimers with lymphotoxin attached to cell surfaces, they usually mediate inflammatory immunostimulatory and antiviral responses, they form lymphoid organ during development and act in control of apoptosis which make them to be tumor suppressor. Immunohistochemistry may also be applied as a confirmatory test for confirmation of the presence of tumor in a particular tissue or sample and to monitor the progress of the treatment administered to the patient.

The use of Enzyme Linked Immunoabsorbent Assay is of great value as well as various monoclonal and polyclonal antibody are employed based on the principle of use of known antibody to detect the present of antigens has been found to facilitate the diagnostic approach, the higher the concentration of the antibody in the assay, the more the presence of tumor in the sample obtained and the higher in the treatment to bring the situation under control. Modern anti-cancer therapies revealed positive response and survival, but the side or adverse effects and high cost frequently leads to discontinuation. Therefore, there is an imperative need of highly effective compounds with tolerable adverse effects that are affordable and at the patient's disposal. Tamoxifen has been used in the treatment of Breast cancer; it has been implicated in many conditions that have practically made it not suitable as reliable therapeutic form of drugs. Due to ongoing progress of the chemotherapeutic agents in cancer management, it remains choice of treatment for breast cancer. However, there is limited finding or no scientific backing on the curative effect. The need of Citrus Limon juice has been attributed to posse's giardiacidal activity, antioxidant activity, pediculicidal activities, and antibacterial activities. Citrus Limon can also be used for douching in sexually active women, while the protective activity in rat urolithiasis model had been proved as well. Despite these activities, limited or no scientific based evidence on anticancer activities of the juice in-vivo using animal model and the need for possible efficay using animal induced model is also encouraged, while the need to establish possible, safety affordable and potent drug for the treatment of breast cancer worldwide is of great value considering the gene or protein expression in the monitoring and evaluation of possible drug effects of Citrus Limon Juice and Tamoxifen on Breast cancer Associated Organs.

\section{Material and methods}

\subsection{Chemicals, Reagents and Equipments}

All the chemicals, reagents, and equipments used in this study are of international standard organization grade stardandardised by ISO and analytical grade without any form of impurities.

\subsection{Citrus Limon fruit Collection}

The Citrus Limon fruit samples of the same species and varieties were collected from a the local farm in Uyo, AkwaIbom, Nigeria within the month of October and December 2016 from the same set of trees in sterilized polythene bags, stored at $4 \mathrm{oC}$ in a refrigerator until use, they were authenticated by Botanist at the Agricultural Biotechnology unit, Derindam Research Institute of Biotechnology, Voucher specimen number DRIB-ABU-005.11 was created for the fruit and deposited in the herbarium. 


\subsection{Acute Toxicity Study of Citrus Limon (L) juice}

Acute toxicity study of Citrus Limon (L) juice was carried out based on Lorke's method [4], LD50 values of the Citrus Limon (L) at 10\%, 20\% and 30\% were $8.88 \%, 17.32 \%$ and $25.98 \%$ and they were considered and used as Low, Middle and High dose respectively.

\subsection{Drugs}

Tamoxifen citrate tablets (Cipla Ltd., Goa 403 722. India).

\subsection{Ethical Clearance on the use of Animals and Management}

Experimental rats used were approved by Animal Care and Use Committee (IAUC) of Derindam Research Institute of Biotechnology Research and Ethical Committee, Nigeria based on the rules guiding the use of laboratory in line with the care of the animals, and the experimental protocol were in strict conformity with the Institute of Laboratory Animals Research (ILAR) guidelines [14]. 120 virgin-female Sprague-Dawley (SD) rats (40 days old) with weight of 180-220g were obtained from the Animal house of the Institute, DRIB. The animals were divided into Ten (10) groups of 12 rats per group. Animals were housed two rats per plastic cages and allowed to acclimatize in standard conditions under a 12 hours light and dark reaction, free access to distilled water and commercialized food throughout the experiment.

\subsection{Preparation Breast cancer (MCF-7) Cell lines}

MCF-7 (Breast cancer) Cell lines were obtained from NCCS, Pune and prepared using modified method of [20]. Cells were cultured in Duilecco's modified Eagle's medium, 10\% Fetal Bovine Serum complete medium supplemented with antibiotics, cells were maintained at $37^{\circ} \mathrm{C}$ in a $5 \% \mathrm{CO} 2$ incubator and the media were changed regularly throughout the experiment. $90 \%$ of the cells were confluent, the medium was removed and the cells washed with Phosphate buffer solution, dead cells were removed by the addition of EDTA to detach the stacked cells. Cells obtained immediately were centrifuged at $1000 \mathrm{rpm}$ for 10 minutes at 4oC, the cells were washed twice with PBS and dispersed.

\subsection{Extractin of Citrus Limon (L) Juice}

Juice was extracted using the method of [15]. Fruit were washed with Distilled water, the juice was extracted manually, by cutting the fruits in halves and carefully squeezing to extract juice using cloth of muslin of 4 fold into the beakers for the administration during the experimental procedures.

\subsection{Breast Cancer / Mammary Tumor Induction}

Mammary Tumor were induced using slight modification method [16]. Experimental animals were anaesthesized using $150 \mathrm{mg} / \mathrm{kg}$ ketamine and $10 \mathrm{mg} / \mathrm{kg}$ of xylazine mixture by injection via intraperitoneal respectively. The injection site was properly cleaned and sterilized with ethanol. The cell suspension, 600000 cells in $300 \mu$ l PBS was drawn into $1 \mathrm{ml}$ TB syringes without needles to minimize damage, lysis and death to the cells. The cell suspension was inoculated subcutaneously into the mammary fat pad (right flank) of the Sprauge- Dawley (SD) rats using a TB syringe with \#26 gauge needle, cell suspension of $300 \mathrm{ul}$ was injected by positioning the needle at $2 \mathrm{~mm}$ posterioly and $2.5 \mathrm{~mm}$ laterally, inserted through the skin and then lowered $5 \mathrm{~mm}$ into the mammary fat pad. The beds of rats were supported with suitable heat lamp to avoid loss of body heat during the procedure. The temperature, breathing and heart rated of animals were monitored closely. The rat were swang backward and over continuously for 30 seconds to generate warmt as this facilitate their breathing rate and they became stabilized shortly after this procedure.

\subsection{Experimental Design for In - vivo Anticancer Study}

One hundred and Twenty Sprague -Dawley rats,40 days old, average body weight 180 -220g were divided into ten (10) groups ( labeled as group 1-10) containing Ten (12) animals per group. Cancer was induced using MCF-7 cell lines [21] in groups 2,7,8,9,10,11 and 12, after twenty one days of development of Breast cancer, animals were treated with various concentrations of Citrus Limon juice and Tamoxifen respectively for Twelve (12) weeks as indicated below:

- Group 1: $\quad$ Control animal fed with feed and water only.

- Group 2: $\quad$ MCF-7 Cell line Induced Breast cancer rats only

- Group 3: $\quad$ Citrus Limon Juice, 8.88\% (Low dose)

- Group 4: $\quad$ Citrus Limon Juice, $17.32 \%$ (Middle dose)

- Group 5: $\quad$ Citrus Limon Juice, 25.98\% (High dose)

- Group 6: Tamoxifen 20mg/kg only

- Group 7: $\quad$ MCF-7 Cell line Induced Breast cancer rats + Citrus Limon Juice, 8.88\% (Low dose) 
- Group 8: $\quad$ MCF-7 Cell line Induced Breast cancer rats + Citrus Limon Juice, 17.32\% (Middle dose)

- Group 9: $\quad$ MCF-7 Cell line Induced Breast cancer rats + Citrus Limonjuice, 25.98\% (High dose)

- Group 10: MCF-7 Cell line Induced Breast cancer rats + Tamoxifen0.2mg/kg

\subsection{Preparation of Tamoxifen doses for Administration}

Tamoxifen was prepared using modified method [17]

\subsection{Tumor Study}

Following the Breast cancer induction, all the animals were monitored on daily baisis for any form of tumour growth and development, areas found to be of abnormal groth in Tumour mass were measured using the formulae of Carisson: $\mathrm{V}=(\mathrm{ab} 2) / 2$ to calculate the following following the measuremt of Length and Breast and witdthof the area of the tumour, where 'a' and 'b'dennotes Length and Breath tumour distance covered or measured by use of the caliper.

\subsection{X-ray Imaging}

Confirmation of the induction of Breast cancer was carried out using the method [18]. Experimental animals were shaved toward the area of mammillary pad and anaesthetized following the induction of tumor, the of X-ray machine a 44 kilvolts for 3 miiliseconds made in Taiwan the advanced radiographs was used to observe the photographs to the presence tumor formed and the location of the Tumor as a confirmatory test the presence of Mammary tumor induced.

\subsection{Sterility Test of Breast cancer (MCF-7) Cell lines}

Sterility test was carried out using the method [19] from the onset to verify the Citrus Limon juice capacity of contamination free, $35 \mathrm{~mm}$ culture dish was plated with MCF-7 cell suspension in $2 \mathrm{ml}$ of DMEM media, cells were allowed to adhere, the Citrus Limon juice were added into the culture dishes and incubated in 5\% CO2 Incubator for 24 hours.

\subsection{Preparation of Cell lines}

MCF-75 Breast cancer cell lines were cultured in DMEM 10\% FBS complete medium using the method [20], 10\% heated inactivated fetal bovine serum was added, antibiotics, and the cells were maintained in 5\% CO2 incubator at 37 degree Celsius and the media were changed regularly throughout the experiment.

\subsection{Protein Expression of Breast Cancer Associated Organ Tumor: Kidney Injury molecule, Rat Liver Cell Membrane Antibody, Lung Tumour Antigen Assay.}

\subsubsection{Rat Kidney Injury molecule (KIM) Assay}

This was carried out using modified method [11] in which Enzyme linked Immuno-absorbent Assay utilized the microplate pre - coated with antibody specific to Kidney Injury Molecule -1, Standard, samples were added to the appropriate microplate wells and combined with the specific antibody, then Horadish-Peroxidase conjugated antibody specific to Kidney Injury Molecule was added to each microplate well and incubated for 1 hour, free component were washed away, Colour development substrate solutions were added to each well in microplate, those well containing Kidney Injury Molecule and horadish peroxidase conjugated antibody appeared Blue in colour and later turned yellow upon the addition of stop solution, the absorbance were measured with microplate reader at wavelength of 450nm, the absorbance measured was directly proportional to the concentration of Kidney Injury Molecule - 1 in the serum samples.

\subsubsection{Rat Liver Cell Membrane Antibody (LCMA) Assay}

This was carried out using modified method [12] in which Enzyme linked Immuno-absorbent Assay utilized the microplate the microplate pre - coated with antibody specific to Liver cell Membrane Antibody, Standard, samples were added to the appropriate microplate wells and combined with the specific antibody, then Horadish-Peroxidase conjugated antibody specific to Liver cell Membrane Antibody was added to each microplate well and incubated for 1 hour, free component were washed away, Colour development substrate solutions were added to each well in microplate, those well containing Liver cell Membrane Antibody and HRP conjugated antibody appeared Blue in colour and later turned yellow upon the addition of stop solution, the absorbance were measured with microplate reader at wavelength of $450 \mathrm{~nm}$, the absorbance measured was directly proportional to the concentration of Liver cell Memebrane Antibody, in the serum samples and it was calculated by comparing the concentration of the Liver cell Memebrane Antibody to the standard curve obtained from the serial dilution of the standard concentration. 


\subsubsection{Rat Lung Tumor Antigen (LTA) Assay}

This was carried out using modified method [13] in which Enzyme linked Immuno-absorbent Assay utilized the microplate the microplate pre - coated with antibody specific to Lung Tumor Antigen, Standard, samples were added to the appropriate microplate wells and combined with the specific antibody, then Horadish-Peroxidase conjugated antibody specific Lung Tumor Antigen was added to each microplate well and incubated for 1 hour, free component were washed away, TMB substrate solutions were added to each well in microplate, those well containing Lung Tumor Antigen and Horadish peroxidase conjugated antibody appeared Blue in colour and later turned yellow upon the addition of stop solution, the absorbance were measured with microplate reader at wavelength of $450 \mathrm{~nm}$, the absorbance measured was directly proportional to the concentration of LTA, in the serum samples and it was calculated by comparing the concentration of the LTA to the standard curve obtained from the serial dilution of the standard concentration.

\section{Results and discussion}

Effect of Citrus Limon juice and Tamoxifen on the Breast Cancer Associated Organ Tumour of MCF-7 cell induced Breast cancer in Sprague dawley rats.

\subsection{Kidney Injury Molecule (KIM)}

Breast Cancer Induced group, BCIR showed high concentration of KIM extremely significant increase and different from the control groups and slightly non-significant increase in the BCIR+CLJ and BCIR+Tamoxifen group, while appered to be non - significant in all the CLJ and Tamoxifen groups as as well as control group meanwhile the BCIR was found to be significant from all the groups at $\mathrm{P}>0.001$.

\subsection{Liver Cell Membrane Antibody (LCMA)}

Protein expression in BCIR revealed very low LCMA concentration and was found to be extremely significant reduced when compared to the BCIR+CLJ, BCIR+Tamoxifen, CLJ alone and Tamoxifen groups as well as control while the BCIR concentration was significantly increase in the CLJ and Tamoxifen alone when compared to the BCIR+CLJ, BCIR+Tamoxifen and control group at $\mathrm{p}>0.005$.

\subsection{Lung Tumour Antigen (LTA)}

was signifancly reduced in the entire treatment groups when compared to the BCIR group and was found to be extremely significant different from BCIR+CLJ and BCIR+Tamoxifen group at $\mathrm{p}>0.005$ while the BCIR only was significantly different from the conrol groups.

Table 1Effect of Citrus Limon juice and Tamoxifen on the Breast Cancer Associated Organ Tumours: Anti Liver Cell membrane Antibody, Kidney Injury Molecule and Lungs Tumour Antigen of MCF-7 cell induced Breast cancer in Sprague Dawley Rats

\begin{tabular}{|c|c|c|c|}
\hline Groups & Anti-LCMA (mg/dl) & KIM (mg/dl) & LTA (mg/dl) \\
\hline Control (Distilled water) & $10.84 \pm 0.431$ & $0.480 \pm 0.041$ & $18.76 \pm 1.596$ \\
\hline BCIR (MCF-7) only & $80.43 \pm 0.098^{\mathrm{a}}$ & $33.56 \pm 1.792^{\text {a }}$ & $300.1 \pm 12.38^{\mathrm{a}}$ \\
\hline Citrus Limon (8.88\%) & $20.92 \pm 0.483^{a}$ & $0.470 \pm 0.035 \mathrm{~ns}$ & $10.38 \pm 0.570^{\mathrm{ns}}$ \\
\hline Citrus Limon (17.32\%) & $32.06 \pm 1.777$ a & $0.412 \pm 0.026^{\mathrm{ns}}$ & $7.200 \pm 0.789 \mathrm{~ns}$ \\
\hline Citrus Limon (25.9\%) & $32.12 \pm 3.459^{a}$ & $0.402 \pm 0.033 \mathrm{~ns}$ & $5.400 \pm 1.351^{\mathrm{b}}$ \\
\hline $0.20 \mathrm{mg} / \mathrm{kg}$ Tamoxifen & $36.79 \pm 1.863^{a}$ & $0.342 \pm 0.011^{\mathrm{ns}}$ & $4.420 \pm 0.821^{b}$ \\
\hline BCIR + C. limon (8.88\%) & $62.44 \pm 5.157$ a & $28.22 \pm 0.787$ a & $213.9 \pm 7.576^{\mathrm{a}}$ \\
\hline BCIR + C. limon $(17.32 \%)$ & $60.22 \pm 1.625^{\mathrm{a}}$ & $23.42 \pm 1.133^{a}$ & $219.9 \pm 4.887^{a}$ \\
\hline BCIR + C. limon $(25.98 \%)$ & $51.10 \pm 2.206^{\mathrm{a}}$ & $17.99 \pm 2.231^{\mathrm{a}}$ & $190.6 \pm 5.391^{\mathrm{a}}$ \\
\hline BCIR + 0.2mg/kg Tamoxifen & $47.44 \pm 2.291^{\mathrm{a}}$ & $0.658 \pm 0.025 \mathrm{~ns}$ & $171.6 \pm 9.647$ a \\
\hline
\end{tabular}

Anti-Liver Cell membrane Antibody (Anti-LCMA), Kidney Injury Molecule (KIM) and Lungs Tumour Antigen (LTA) 


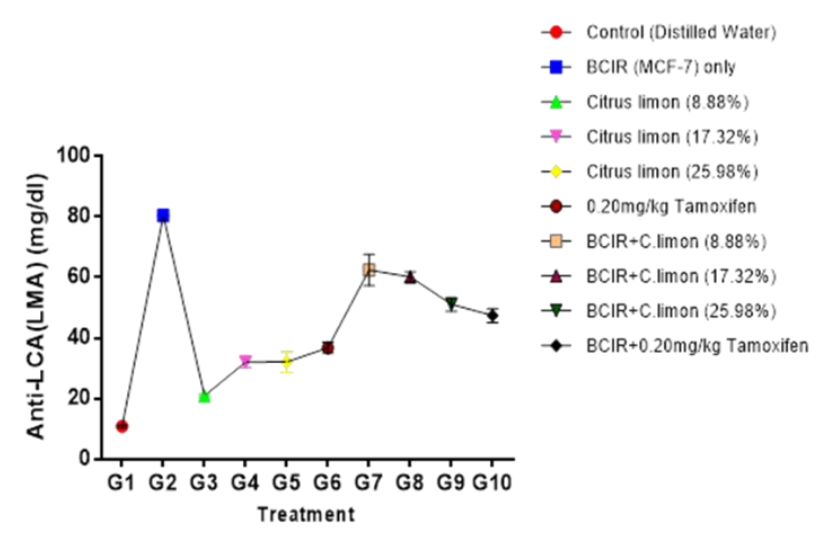

Figure 1Anti - Liver Cell Memebrane Antibody Expression

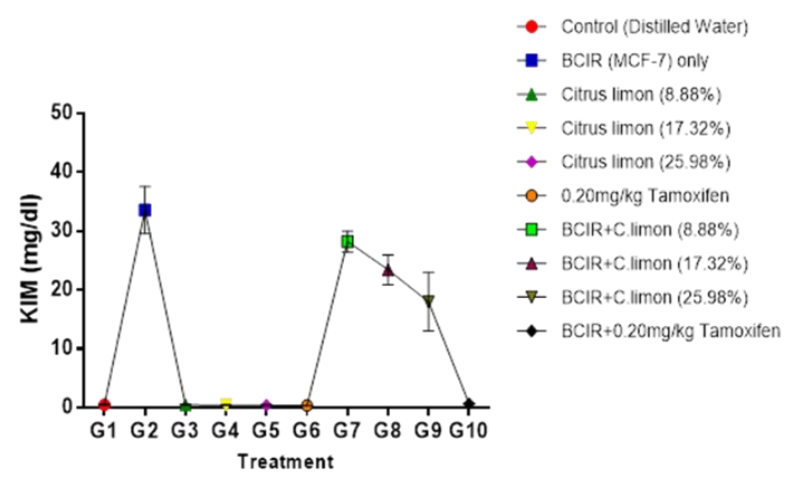

Figure 2 Kidney Injury Molecule Expression

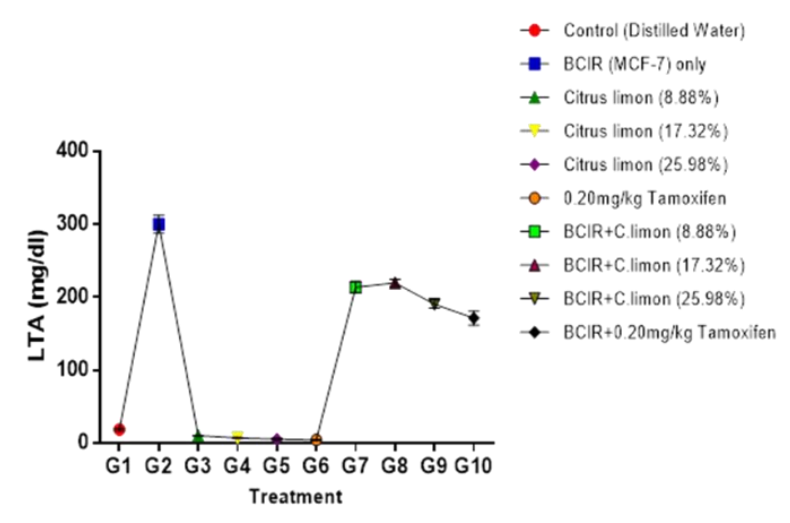

Figure 3 Lungs Tumour Antigen Expression

\section{Discussion}

Series of conventional therapies have been employed and utilized in orthodox, traditional and herbal medicine, yet no clear-cut possible cure have been identified.

Clinical wise, the use of chemotherapy has produced slight significant relieve but the end product still result in dead or irreversible loss of life, even when it has caused the patient huge loss of money, fortune and profile lost in the society. Radiotherapy as another form of cure have been employed in many cases, they appeared working initially but at later end causes pain, wasteful and loss of life and general lost in the society. (www.cancer.org) 
According to [2], variety of conventional therapies for cancer based on chemotherapy, radiotherapy and surgery are limited in efficacy. Most current cancer chemotherapy regimens are normally associated with very high significant levels of toxicity and drug resistance.

Manufacturing of drugs employed in the treatment of breast cancer has not been easy as it involve huge capital intensive, man-power and exploration of ideas from scientist, medical expert across the globe.

Finding easily available alternative for the treatment, cure, prevention and management will surely pave ways for targeted different types of cancer and making the treatment easy thereby reducing the mortality rate of cancer in our society. Major challenges to reduce breast cancer burden is to develop highly effective alternative drugs specifically for breast cancer with minimal side effects or no side effect on the patients

Kidney injury molecules (KIM) as an putative epithelial cell adhesion molecule found in the group of immunoglobulin which is upregulated during injury to renal cells, kidney as an excretory organ played vital role in cancer study and chemotherapy, drug designed or administered to the patient after activities, the waste product are excreted out of the body via kidney, renal cells of the glomerulus, convulated tubules are major cells that upregulate the outlet of urine, when injury resulted from the damages from the drug on the cells have direct influence on the kidney and kidney injury molecule concentration showed that the breast cancer induced group revealed high immunological expression indicating renal injury and impairment which lead to increase in the concentration value of KIM extremely above the normal range, meanwhile in the breast cancer induced group post-treated with Citrus Limon juice at different concentration, the KIM concentration revealed low immunologic expression as the concentration increases thereby resolving the injured renal cell into the normal shape in similar manner with the Tamoxifen reference drugs while the other non-breast cancer induced groups were normal level of KIM without any form of renal injury [5] and [6]

Anti-lung tumor antigen (LTA) is protein belonging to the family of tumor necrosis factor, cytokine like in nature produced by lymphocytes, it is inducible, secreted and form anetertotrimers with lymphotoxin attached to cell surfaces, they usually mediate inflammatory immune-stimulatory and antiviral responses, they form lymphoid organ during development and act in control of apoptosis which make them to be tumor suppressor like genes or protein during the experiment.

Lungs played very important role in exchange of gases in and out of the body MCF-7 cell induced breast cancer in rats caused injury and inflammatory responses to other associated organ, thereby making breathing difficult due to loss of immunity in the breast cancer induced group, the LTA concentration increases by revealing high immunological expression as compared to the breast cancer induced groups post-treated with Citrus Limon at various concentration which was found to be reduced with low expression immunologically and gradually displayed dose dependent activities due to possible therapeutic effect of Citrus Limon on Tamoxifen reference drug while LTA concentration in the other non-control and non-breast cancer group were within the normal range [12].

Liver cell membrane antigen (LCMA) is the protein of parenchymal and non-parenchymal cell of the liver that are expressed or produced during disturbance to the liver cells or injury. LCMA concentration in the membrane of the amount of injuries agent in form of immunoglobulin that have direct effect on the liver cells and this help in chemotherapy to measure the drug response to the injury inflicted on the liver in detoxification of oxygen, breast cancer induced group revealed high concentration and immunological expression of LCMA due to MCF-7 cell line induced cancer while the post-treated group of breast cancer induced group with Citrus Limon at various concentration showed dose response effect of the treatment on the induced cancer group similarly with the Tamoxifen reference drug groups while non-breast cancer induced group appear normal with very low or no concentration giving rise to low immunologic expression of LCMA with references to response of anticancer properties the Citrus Limon juice and Tamoxidfen exhibited simultaneously, though the expressions in these protein were significantly demonytrated [3].

\section{Conclusion}

Findings from this study had shown that immunogenic expression of Breast Carcinoma-Associated Organ tumors such as Kidney injury molecules (KIM), Anti lung tumor antigen (LTA) and Liver cell membrane antigen (LCMA) were significantly increased in concentration in all the Breast cancer induced groups and the reverse in immunogenic expression was obtained in post administered experimental animals with various doses of Citrus Limon juice and Tamoxifen reference drug, hence the use of Breast Carcinoma-Associated Organ makers have shown possible, significant evaluating and monitoring capacities on chemotherapeutic activities of the Citrus Limon Juice in a relevant manner with that of Tamoxifen. 


\section{Compliance with ethical standards}

\section{Acknowledgments}

Authors appreciate and acknowledge laboratory and computer staff of the department where these experiments were carried out.

\section{Disclosure of conflict of interest}

All authors declare no conflicts of interest associated with this manuscript.

\section{Statement of informed consent}

The authors have no conflicts of interest to declare.

\section{Authors' contributions}

This work was carried out in collaboration between all authors. Author OSA designed, supervised the study and undertook the final editing of the paper. Author NSA and OOE wrote the protocols, handling of the experimental animal induction, carried out laboratory analysis and performed the statistical analysis under the close supervision of Author OSA. All authors read and approved the final manuscript.

\section{Statement of data availability}

The datasets generated during the analysis used to support our findings of this study are available from the first and corresponding authors on reasonable requests.

\section{References}

[1] American Cancer society. 2016.

[2] Hsiao WL, Liu L. The role of traditional Chinese herbal medicines in cancer therapy from TCM theory to mechanistic insights. PlantaMedica. 2010; 76(11): 1118-1131.

[3] IG Mcfarlane, CGMcSorley, JE Hegarty. Antibodies to liver-specific protein predict outcome of treatment withdrawal in autoimmune chronic active hepatitis," The Lancet. 1984; 2(8409): 954-956.

[4] Lorke DA. A new approach to practical acute toxicity testing. Arch Toxicol. 1983; 54:27-87.

[5] TakaharuIchimural, Joseph V. Bonventres, Veronique Bailly, Henry Wei, Catherine A. Hession, Richard L. Cate and Michelle Sanicolas. Viel. 1998; 273(7): 4135-4142.

[6] Van Wynberge L, Vierling P, Lampel A. Metastasis of a breast carcinoma in a many renal cell carcinoma: Current Urology. 2004; 35(06): 505-507.

[7] Bale R, Putzer D, Schullian P. Local Treatment of Breast Cancer Liver Metastasis. Cancers. 2019; 11(9): 13-41.

[8] Liu W, Peng JF, Tang MJ. Individualized Treatment Analysis Of Breast Cancer With Chronic Renal Failure. OncoTargets and therapy. 2019; 12: 7767-7772.

[9] Jin L, Han B, Siegel E, Cui Y, Giuliano A, Cui X. Breast cancer lung metastasis: Molecular biology and therapeutic implications. Cancer biology \& therapy. 2018; 19(10): 858-868.

[10] SongJ, Yu J, Prayogo GW, Cao W, Wu Y, Jia Z, Zhang A. Understanding kidney injury molecule 1: a novel immune factor in kidney pathophysiology. American journal of translational research. 2019; 11(3): 1219-1229.

[11] Han WK, Bailly V, Abichandani R, Thadhani R, Bonventre JV. Kidney injury molecule-1 (KIM-1): A novel biomarker for human renal proximal tubule injury. Kidney Int. 2002;62:237-244.

[12] Toda G, Ikeda Y, Hashimoto N, Yamazaki M, Torii M, Oka H. Liver cell membrane antibody detected by protein A and isolated rabbit liver plasma membrane in sera of patients with chronic liver diseases. Clinical and experimental immunology. 1983; 54(3): 661-670. 
[13] Zhang Z, Wodzak M, Belzile O, Zhou H, Sishc B, Yan H, Stojadinovic S, Mason RP, Brekken RA, Chopra R, Story MD, Timmerman R, Saha D. Effective Rat Lung Tumor Model for Stereotactic Body Radiation Therapy. Radiation research. 2016; 185(6): 616-622.

[14] ILAR (Institute of Laboratory Animal Research), Commission on life science; National Research Council. 1996.

[15] SSF Janati, HR Beheshti J, Feizy, NK Fahim. Chemical composition of lemon (Citrus Limon) and peels its consideration as animal Food". Gida. 2012; 37(5): 267-271.

[16] Russo j, Gusterson B, Rogers A, Russo I, Wellings S, Van ZwietenMj. Comparative study of human and rat mammary tumorigenesis. Laboratory investigation. 1990; 62(3): 244-278.

[17] Turner PV, Brabb T, Pekow C, Vasbinder MA. Administration of substances to laboratory animals: routes of administration and factors to consider. Journal of the American Association for Laboratory Animal Science: JAALAS. 2011; 50(5): 600-613.

[18] Workman P, Aboagye EO, Balkwill F, Balmain A, Bruder G, Chaplin DJ, Kelland LR. Guidelines for the welfare and use of animals in cancer research. British journal of cancer. 2010; 102(11): 1555-1577.

[19] Firdhouse J, Lalitha P. Apoptotic efficacy of biogenic silver nanoparticles on human breast cancer MCF-7 cell lines. Progress in biomaterials. 2015; 4(2-4): 113-121.

[20] Vantangoli MM, Madnick SJ, Huse SM, Weston P, Boekelheide K. MCF-7 Human Breast Cancer Cells Form Differentiated Microtissues in Scaffold-Free Hydrogels. PloS one. 2015; 10(8): e0135426.

[21] RoghayehAbbasalipourkabir, ArashDehghan, ArefSalehzadeh, FatemehShamsabadi and RasedeeAbdulah. Induction of mammary gland tumour in female Sprawgue-Dawley rats with LA7 Cells. African Journal of Biotechnology. 2010; 9(28): 4491-4498. 